\title{
Nonresonant Cross-Section, Fano-Shape Parameter and Correlation Coefficient for the Photoionization of 2s2p $\left({ }^{1} \mathbf{P}^{\circ}\right)$ in Heliumlike
}

\author{
Aliou Niane, Ndeye Arame Boye Faye, Ababacar Sadikh Ndao, and Almamy Konte \\ Laboratory Atoms Lasers, Department of Physics, Cheikh Anta Diop University, 5005 Dakar, Senegal \\ Correspondence should be addressed to Ndeye Arame Boye Faye, arame@ucad.sn
}

Received 13 August 2010; Revised 1 November 2010; Accepted 6 December 2010

Academic Editor: Igor Bray

Copyright (C) 2011 Aliou Niane et al. This is an open access article distributed under the Creative Commons Attribution License, which permits unrestricted use, distribution, and reproduction in any medium, provided the original work is properly cited.

\begin{abstract}
The nonresonant cross-section, Fano-shape parameter $q$ and coefficient parameter $\rho^{2}$ have been calculated for the photoionization of helium and heliumlike under the $n=2$ threshold of the residual ions. Dipole matrix elements which correspond to the transitions from $1 s^{2}\left({ }^{1} S^{\mathrm{e}}\right)$ to ${ }_{2}(0,1)_{2}^{+1} \mathrm{P}^{\circ}$ state and from ${ }_{2}(0,1)_{2}^{+}$state to continuum state $1 \mathrm{sep}\left({ }^{1} \mathrm{P}^{\circ}\right)$ were determined by choosing, for the ground state, the six-parameter Hylleraas wave function including electron correlation effects and a completely screened Coulomb wave function for the continuum. Energies and widths have been determined in the diagonalization method. The results are compared with available results obtained by other theoretical approaches.
\end{abstract}

\section{Introduction}

Doubly excited states of helium were first studied experimentally in the pioneering work of Madden and Codling [1] with the availability of synchrotron radiation. Madden and Codling [1] observed the two ${ }^{1} \mathrm{P}^{\circ}$ series of transitions converging to the $\mathrm{He}^{+}(n=2)$ ionization limit, using a photoabsorption technique. Thereafter, many experimental studies [2-8] were carried out on the doubly excited states of helium, and much effort has been done in photoionization that leaves the $\mathrm{He}^{+}$ion in an excited state. Single-photon double-excitation experiments of Domke et al. [9-11] using synchrotron radiation combined with high-resolution monochromators, and even further improved monochromators in the experiment of Schulz et al. [12] made it possible to reveal for the first time several additional Rydberg series. Domke et al. [9] resolved for the first time the third Rydberg series, $2 \mathrm{p} n \mathrm{~d}$, below the $n=2$ ionization threshold. Theoretically, various methods were developed to deal with doubly excited states of helium, including configurationinteraction (CI) methods [13-15], hyperspherical coordinate methods [16-23], close-coupling approximations [24-26], R-matrix methods [27-32], complex coordinate method [33-36], Feshbach projection formalism [37-40], and the diagonalization method [41-47]. A detailed review was recently performed by Tanner et al. [48].

Since the identification of doubly excited states of $\mathrm{He}$ by Madden and Codling [1], it has been well established that these states cannot be adequately described in the conventional independent-particle model. According to the conventional model, the two electrons are described by using quantum numbers $n_{i}, l_{i} L, S, \pi, M_{L}$, and $M_{S}$, within the $L S$ coupling, with $n_{i}$, the principal quantum number of electron $i$. Because of the strong configuration, these quantum numbers are not sufficient to identify the states. A different classification scheme for doubly excited states of $\mathrm{He}$ is used depending on authors. In the classification introduced by Moccia and Spizzo [13] and Lin [18], states are denoted by ${ }_{n}(K, T)_{N}^{A}{ }^{2 S+1} L^{\pi}$ where $L, S$, and $\pi$ have their usual meaning, $N$ and $n$ are the principal quantum numbers of the inner and outer electrons, respectively, and $K, T$, and $A$ are correlation quantum numbers. $K$ ranges from $N-1-T, N-3-$ $T \cdots-(N-1-T)$, and $T=0$ or 1 for ${ }^{1} \mathrm{P}^{\circ}$ states. Both $K$ and $T$ describe the angular correlation between the two electrons, while $A=+1,-1,0$ or denoted by "+", "-", " 0 " measures the radial correlation [18]. The doubly excited states-basis (DESB) functions proposed by Moccia and Spizzo [13] 
represent the first-order approximation of these states. Through the analysis of electron correlations, a new set of quantum numbers were introduced to replace the quantum numbers used in the independent particle model. There is also an abbreviated classification $N, K_{n}$ as introduced by Zubek et al. [6]. There are three ${ }^{1} \mathrm{P}^{\circ}$ series converging to the $n=2$ threshold: $2,0_{n}$ with $T=1$ and $A=$ $+1,2,1_{n}$ with $T=0$ and $A=-1$, and $2,-1_{n}$ with $T=0$ and $A=0$. These sates correspond respectively to $(\mathrm{sp}, 2 n+),(\mathrm{sp}, 2 n-)$, and $(2 \mathrm{p}, n \mathrm{~d})$ in the old classification scheme.

Until recently, doubly excited states lying the $n=$ 2 received great attention. In their work, Yuan et al. [49] reported Fano profile parameters $q$ of doubly excited states ${ }_{n}(1,0)_{2}^{+1} S^{\mathrm{e}},_{n}(1,0)_{2}^{+1} D^{\mathrm{e}}$, and ${ }_{n}(0,1)_{2}^{+1} \mathrm{P}^{\circ}$ as functions of the momentum transfer squared $K^{2}$. Fan and Leung [50], presented a detailed characterization of the absolute generalized oscillator strength (GOS) profile of the $\mathrm{He} 1 \mathrm{~s}^{2} \rightarrow$ $2 \mathrm{~s} 2 \mathrm{p}$ transition by using electron energy loss spectroscopy (EELS) at $2.5 \mathrm{keV}$ impact energy, and they illustrated the intricate changes in the Fano parameters as functions of the momentum transfer. Prince et al. [51] measured the photoabsorption cross-section and ion-yield spectra of $\mathrm{He}$ in the region of the double-excitation resonances below the $n=2$ threshold. Very recently, more than thirty years after the experimental work of Carroll and Kennedy [52], Scully et al. [53] measured the absolute cross-section for resonant double photoexcitation of $\mathrm{Li}^{+}$followed by autoionization in the photon energy range from $148 \mathrm{eV}$, just below the ${ }_{2}(1,0)_{2}^{+}$ resonance to $198 \mathrm{eV}$.

In this present work, we focused on the determination of Fano-Shape parameter or profile index $q$, correlation coefficient $\rho^{2}$, and nonresonant cross-section $\sigma_{b}$ in the photoionization of helium and heliumlike with charge $Z=$ $3,6,8$, and 10 from the ground state to the ${ }_{2}(0,1)_{2}^{+1} \mathrm{P}^{\circ}$ state. Until now, only few theories have calculated $q$ and $\rho^{2}$ for helium and heliumlike $\mathrm{Li}^{+}$and $\mathrm{C}^{4+}$, and in addition, experiments on the photoionization of heliumlike positive ions are very scarce. Here we extend calculations to heliumlike $\mathrm{N}^{5+}, \mathrm{O}^{6+}$, and $\mathrm{Ne}^{8+}$. We also present energies and widths, calculated in the diagonalization method, which we have already used in our previous works [43-47].

\section{Theory and Calculations}

In Fano's theory [58], describing photoabsorption in the vicinity of an autoionizing resonance, which coincides in energy with a continuum, Fano profile consists of a constructive and a destructive interaction of the direct photoionization with the indirect photoionization via doubly excited or autoionizing states. So, according to Fano, the total CrossSection for the case of an isolated resonance interacting with one or more continuum states can be parameterized as follows:

$$
\sigma(E)=\sigma_{a} \frac{(q+\varepsilon)^{2}}{\left(1+\varepsilon^{2}\right)}+\sigma_{b}, \quad \text { with } \varepsilon=2 \frac{E-E_{r}}{\Gamma},
$$

where $E_{r}$ is the resonance energy, $\Gamma$ the width, $\varepsilon$ indicates the deviation of the incident photon energy $E$ from the resonance energy $E_{r}, \sigma_{a}$ and $\sigma_{b}$ represent the Cross-Section corresponding, respectively, to transitions to states of the continuum that do and do not interact with the discrete autoionizing state and finally $q$ which characterizes the line profile representing the ratio of the dipole matrix element of a transition to a discrete state to that of a transition to the continuum, which interacts with the discrete state. In the present case, the Rydberg series converging to the $n=2$ threshold interact with the continuum above the $n=1$ ionization threshold. In this theory [58], the profile index $q$ can vary from $|q| \rightarrow \infty$, when the transition probability into the discrete state is dominant (Lorentzian profile), to $q \rightarrow 0$, when the transition probability into the discrete state is much smaller than that into the continuum, leading to a symmetric decrease of the Cross-Section to a minimum value ("window" resonance). Fano and Cooper [59] express the total Cross-Section in terms of the nonresonant CrossSection $\sigma_{o}$ and the profile index $q$ and correlation coefficient $\rho^{2}$ as follows:

$$
\sigma(E)=\sigma_{o}\left[\rho^{2} \frac{(q+\varepsilon)^{2}}{\left(1+\varepsilon^{2}\right)}+1-\rho^{2}\right],
$$

where $\rho^{2}$ is a measure of the strength of the resonance.

In the diagonalization approximation, the final state of the ion + photoelectron system is expanded in the subspaces of closed and open channels as follows:

$$
\Psi_{E j}\left(r_{1}, r_{2}\right)=\hat{A} \sum \Psi_{k}\left(r_{1}\right) U_{k j}\left(E, r_{2}\right)+\sum \Lambda_{\mu}(E) \Phi_{\mu}\left(r_{1}, r_{2}\right),
$$

where $\hat{A}$ is the operator of antisymmetrisation, $k$ represents a set of quantum numbers that characterize the ion + photoelectron system in the subspace of open channels; $U_{k j}\left(E, r_{2}\right)$ is an unknown function describing the motion of the photoelectron and $\Psi_{k}\left(r_{1}\right)$ is the eigenfunction of the residual ion.

The determination of the function $\Psi_{E j}\left(r_{1}, r_{2}\right)$ is equivalent to the calculation of the coefficients $\Lambda_{\mu}(E)$ and $U_{k j}\left(E, r_{2}\right)$. Detailed calculations of these coefficients have been reported by $[41,42]$. The functions $\Phi_{\mu}\left(r_{1}, r_{2}\right)$ are obtained by unitary transformation of the Hamiltonian in the subspace of closed channels which are the autoionizing states:

$$
\Phi_{\mu}\left(r_{1}, r_{2}\right)=\widehat{A} \sum_{k l} \alpha_{\mu}(k, l) \Phi_{k}\left(r_{1}\right) \Phi_{l}\left(r_{2}\right) .
$$

With the diagonalization condition:

$$
\left\langle\Phi_{\mu}\left(r_{1}, r_{2}\right)|H| \Phi_{\nu}\left(r_{1}, r_{2}\right)\right\rangle=E_{\mu} \delta_{\mu \nu}
$$

$H$ is the Hamiltonian operator; the coefficients $\alpha_{\mu}(k, l)$ which are the statistical weight of the autoionizing states are found by solving the system of linear algebraic equations:

$$
\sum_{\nu}\left[\left(E_{\mu}-E_{0}\right) \delta_{\mu \nu}-\left\langle\chi_{\mu}|V| \chi_{\nu}\right\rangle\right] \alpha_{\nu}=0,
$$

where $E_{\mu}$ is the energy of the autoionizing level $\mu ; V$ is the operator of electrostatic interaction between electrons; 
TABLE 1: Results on energy $E(\mathrm{eV})$, width $(\mathrm{eV})$, nonresonant cross-section $\sigma_{0}\left(10^{-18} \mathrm{~cm}^{2}\right)$, correlation coefficient $\rho^{2}$, and profile index $q$ for the photoionization of $\mathrm{He}$ from $1 s^{2}\left({ }^{1} S^{e}\right)$ to ${ }_{2}(0,1)_{2}^{+1} \mathrm{P}^{\circ}$ state. Present results are compared with experimental works.

\begin{tabular}{lcccccc}
\hline & Present work & Madden and Codling [1] & Domke et al. [11] & Morgan and Ederer [3] & Schulz et al. [12] & Kossmann et al. [5] \\
\hline$E$ & 60.350 & $60.123 \pm 0.015$ & 60.147 & $60.151 \pm 0.010$ & 60.1503 & $60.133 \pm 0.015$ \\
$\Gamma$ & 0.0375 & $0.038 \pm 0.004$ & 0.037 & $0.038 \pm 0.002$ & 0.0376 & $0.038 \pm 0.001$ \\
$\sigma_{0}$ & 1.491 & - & - & $1.354 \pm 0.030$ & - & $1.376 \pm 0.027$ \\
$\rho^{2}$ & 0.999 & 1.0 & - & 1.0 & - & -2.73 \\
$q$ & -2.86 & $-2.80 \pm 0.25$ & $-2.75 \pm 0.01$ & $-2.55 \pm 0.16$ & $-2.75 \pm 0.05$ \\
\hline
\end{tabular}

TABle 2: Results on energy $E(\mathrm{eV})$, width $(\mathrm{eV})$, nonresonant Cross-Section $\sigma_{0}\left(10^{-18} \mathrm{~cm}^{2}\right)$, correlation coefficient $\rho^{2}$, and profile index $q$ for the photoionization of $\mathrm{He}$ from $1 \mathrm{~s}^{2}\left({ }^{1} S^{e}\right)$ to ${ }_{2}(0,1)_{2}^{+1} \mathrm{P}^{\circ}$ state. Present results are compared with theoretical works.

\begin{tabular}{|c|c|c|c|c|c|c|c|}
\hline & Present work & $\begin{array}{c}\text { Altick and } \\
\text { Moore [54] }\end{array}$ & $\begin{array}{c}\text { Burke and } \\
\text { McVicar [55] }\end{array}$ & Zhou [26] & $\begin{array}{c}\text { Bhatia and } \\
\text { Temkin [38] }\end{array}$ & $\begin{array}{l}\text { Sanchez and } \\
\text { Martin [56] }\end{array}$ & $\begin{array}{l}\text { Wintgen and } \\
\text { Delande [36] }\end{array}$ \\
\hline$E$ & 60.350 & 60.340 & 60.269 & 60.154 & 60.1901 & 60.156 & 60.1466 \\
\hline$\Gamma$ & 0.0375 & 0.0365 & 0.0438 & 0.0378 & 0.0363 & 0.0383 & 0.03736 \\
\hline$\sigma_{0}$ & 1.491 & - & 1.415 & 1.4011 & 1.3865 & 1.374 & - \\
\hline$\rho^{2}$ & 0.999 & - & - & 0.9984 & - & - & - \\
\hline$q$ & -2.86 & -2.97 & -2.59 & -2.73 & 2.8521 & -2.83 & -2.77 \\
\hline
\end{tabular}

$E_{0}$ is the energy eigenvalue of the zero-order Hamiltonian corresponding to the eigenfunctions defined by:

$$
\chi_{v}=\widehat{A}\left[\Phi_{k}\left(r_{1}\right) \Phi_{l}\left(r_{2}\right)\right]
$$

The partial amplitude that describes the formation of the residual ion and photoelectron in a definite state has been defined by the following expression:

$$
\begin{array}{r}
T_{\mathrm{j}}(E)=\left\langle\varphi_{\mathrm{j}}(E)|D| \Psi_{i}\left(r_{1}, r_{2}\right)\right\rangle+\frac{q+i}{\varepsilon-i}\left\langle\Phi_{\mu}\left(r_{1}, r_{2}\right)|V| \varphi_{\mathrm{j}}(E)\right\rangle \\
\times \frac{\sum_{k}\left\langle\Phi_{\mu}\left(r_{1}, r_{2}\right)|V| \varphi_{k}(E)\right\rangle\left\langle\varphi_{k}(E)|D| \Psi_{i}\left(r_{1}, r_{2}\right)\right\rangle}{\sum_{k}\left|\left\langle\Phi_{\mu}\left(r_{1}, r_{2}\right)_{\mu}|V| \varphi_{k}(E)\right\rangle\right|^{2}} .
\end{array}
$$

In (8), $\Psi_{i}\left(r_{1}, r_{2}\right)$ is the initial state and $\varphi_{j}(E)$ is the wave function of the continuous spectrum in the channel $j$, without resonance interference; $D$ is the dipole momentum operator; $q$ is the profile index of the resonance and $\varepsilon$, the relative deviation from the resonance, are defined by the equations:

$$
\begin{gathered}
\varepsilon=\frac{\left(E-E_{\mu}\right)}{(1 / 2) \Gamma_{\mu}^{\mathrm{tot}}} \\
q=\frac{\left\langle\Phi_{\mu}\left(r_{1}, r_{2}\right)|D| \Psi_{i}\left(r_{1}, r_{2}\right)\right\rangle}{\pi \sum_{k}\left|\left\langle\Phi_{\mu}\left(r_{1}, r_{2}\right)|V| \varphi_{k}(E)\right\rangle\right|\left\langle\varphi_{k}(E)|D| \Psi_{i}\left(r_{1}, r_{2}\right)\right\rangle} .
\end{gathered}
$$

With $\Gamma_{\mu}^{\text {tot }}$ the total width:

$$
\Gamma_{\mu}^{\mathrm{tot}}=2 \pi \sum_{j}\left|\left\langle\Phi_{\mu}\left(r_{1}, r_{2}\right)|V| \varphi_{j}(E)\right\rangle\right|^{2} .
$$

In the diagonalization approximation, the total CrossSection defined by Fano [58] can be expressed with the total amplitude by

$$
\sigma(E)=\sigma_{a} \frac{(q+\varepsilon)^{2}}{\left(1+\varepsilon^{2}\right)}+\sigma_{b}=\sum_{j}\left|T_{D}^{j}\right|^{2} .
$$

With $\sigma_{a}$ and $\sigma_{b}$ representing the Cross-Section corresponding, respectively, to transitions to states of the continuum that do and do not interact with the discrete autoionizing state and defined as follows:

$$
\begin{gathered}
\sigma_{a}=\frac{\left|\left\langle\Phi_{\mu}\left(r_{1}, r_{2}\right)|V| \varphi_{k}(E)\right\rangle\left\langle\varphi_{k}(E)|D| \Psi_{i}\left(r_{1}, r_{2}\right)\right\rangle\right|^{2}}{\left|\left\langle\Phi_{\mu}\left(r_{1}, r_{2}\right)|V| \varphi_{k}(E)\right\rangle\right|^{2}}, \\
\sigma_{b}=\left|\left\langle\varphi_{k}(E)|D| \Psi_{i}\left(r_{1}, r_{2}\right)\right\rangle\right|^{2}-\sigma_{a} .
\end{gathered}
$$

The nonresonant Cross-Section is thus defined by:

$$
\sigma_{o}=\left|\left\langle\varphi_{k}(E)|D| \Psi_{i}\left(r_{1}, r_{2}\right)\right\rangle\right|^{2} .
$$

The coefficient correlation can be expressed by:

$$
\rho^{2}=\frac{\sigma_{a}}{\sigma_{a}+\sigma_{b}}
$$

In the present work, we consider for the ground state, the six-parameter Hylleraas wave function including electron correlation effects, and as the emitted electron is in the field of the residual hydrogenic ion, the continuum is represented by a completely screened Coulomb wave function. Energies of resonant states are determined in the framework of diagonalization approximation which has been detailed and discussed earlier [41-47]. Particular interest of the diagonalization method is to take into account the coupling between closed and open channels in terms of perturbation theory and to neglect the indirect coupling of resonant states 
TABLE 3: Results on energy $E(\mathrm{eV})$, width $(\mathrm{eV})$, nonresonant Cross-Section $\sigma_{0}\left(10^{-18} \mathrm{~cm}^{2}\right)$, correlation coefficient $\rho^{2}$, and profile index $q$ for the photoionization of $\mathrm{Li}^{+}$from $1 s^{2}\left({ }^{1} S^{e}\right)$ to ${ }_{2}(0,1)_{2}^{+1} \mathrm{P}^{0}$ state. Present results are compared with theoretical and experimental works.

\begin{tabular}{|c|c|c|c|c|c|c|c|}
\hline & Present Work & $\begin{array}{c}\text { Carroll Kennedy } \\
\text { [52] Exp }\end{array}$ & $\begin{array}{l}\text { Scully et al. } \\
\text { [53] Exp }\end{array}$ & $\begin{array}{l}\text { Diehl et al. } \\
\text { [57] Exp }\end{array}$ & Zhou [26] & $\begin{array}{c}\text { Bhatia and } \\
\text { Temkin [38] }\end{array}$ & $\begin{array}{l}\text { Sanchez and } \\
\text { Martin [56] }\end{array}$ \\
\hline$E$ & 150.642 & $150.29 \pm 0.05$ & $150.31 \pm 0.03$ & $150.31 \pm 0.02$ & 150.260 & 150.247 & 150.295 \\
\hline$\Gamma$ & 0.0621 & $0.075 \pm 0.25$ & $0.057 \pm 0.03$ & $0.066 \pm 0.09$ & 0.0648 & 0.0593 & 0.0622 \\
\hline$\sigma_{0}$ & 0.8910 & - & - & - & 0.5759 & - & 0.573 \\
\hline$\rho^{2}$ & 1.000 & - & - & - & 1.009 & - & - \\
\hline$q$ & -1.53 & $-1.5( \pm 0.3,0.5)$ & $-2.17 \pm 0.04$ & -1.96 & -2.199 & & -2.20 \\
\hline
\end{tabular}

TABle 4: Results on energy $E(\mathrm{eV})$, width $(\mathrm{eV})$, nonresonant CrossSection $\sigma_{0}\left(10^{-18} \mathrm{~cm}^{2}\right)$, correlation coefficient $\rho^{2}$, and profile index $q$ for the photoionization of $\mathrm{C}^{4+}$ from $1 \mathrm{~s}^{2}\left({ }^{1} S^{e}\right)$ to ${ }_{2}(0,1)_{2}^{+1} \mathrm{P}^{0}$ state. Present results are compared with theoretical works.

\begin{tabular}{lcc}
\hline & Present work & Zhou [26] \\
\hline$E$ & 666.960 & 666.287 \\
$\Gamma$ & 0.1046 & 0.0914 \\
$\sigma_{0}$ & 0.1491 & 0.1298 \\
$\rho^{2}$ & 1.000 & - \\
$q$ & -1.20 & -1.74 \\
\hline
\end{tabular}

Table 5: Present results on energy $E(\mathrm{eV})$, width $(\mathrm{eV})$, nonresonant Cross-Section $\sigma_{0}\left(10^{-18} \mathrm{~cm}^{2}\right)$, correlation coefficient $\rho^{2}$, and profile index $q$ for the photoionization of $\mathrm{N}^{5+}, \mathrm{O}^{6+}$, and $\mathrm{Ne}^{8+}$ from $1 \mathrm{~s}^{2}\left({ }^{1} S^{e}\right)$ to ${ }_{2}(0,1)_{2}^{+1} \mathrm{P}^{\circ}$ state.

\begin{tabular}{lccc}
\hline & $\mathrm{N}^{5+}$ & $\mathrm{O}^{6+}$ & $\mathrm{Ne}^{8+}$ \\
\hline$E$ & 919.943 & 1214.293 & 1925.433 \\
$\Gamma$ & 0.1125 & 0.1188 & 0.1282 \\
$\sigma_{0}$ & 0.0595 & 0.0584 & 0.0270 \\
$\rho^{2}$ & 0.999 & 1.000 & 0.999 \\
$q$ & -1.22 & -1.25 & -1.38 \\
\hline
\end{tabular}

through the open channels. The determination of CrossSection, $q$, and coefficient parameter $\rho^{2}$ is carried out by calculating the dipole matrix elements which correspond to the transitions from $1 s^{2}\left({ }^{1} S^{\mathrm{e}}\right)$ to ${ }_{2}(0,1)_{2}^{+1} \mathrm{P}^{\circ}$ state and from ${ }_{2}(0,1)_{2}^{+1} \mathrm{P}^{\circ}$ state to continuum state $1 \operatorname{sep}\left({ }^{1} \mathrm{P}^{\circ}\right)$.

\section{Results and Discussion}

In Table 1, present results on helium are compared with the experimental works of Madden and Codling [1], Schulz et al. [12], Kossmann et al. [5], and Domke et al. [11]. Present results are in good agreement with these experiments. Our profile index is close to the experimental result of Madden and Codling [1] and is in good agreement with the other experimental values. Energies and widths presented are also in good agreement. For the nonresonant Cross-Section, our result is close to the only available one, the value of Kossmann et al. [5].

Concerning the coefficient correlation, the value $\rho^{2}=$ 0.999 (close to unit) we have found is in very good agreement with the experimental ones and agrees with the fact that when there is only one open channel, the part of the Cross-Section $\sigma_{b}$ corresponding to transitions to states of the continuum that do not interact with the discrete autoionizing state is negligible compared to $\sigma_{a}$. This value agreed with the value $\rho^{2}=1$ assumed by the work of Fano and Cooper [59] for a Rydberg series interacting with one continuum. In Table 2, comparison is made with theoretical results of Altick and Moore [54] using configuration interaction method, Burke and McVicar [55] using the close coupling method, Sanchez and Martin [56] using variational method with $L^{2}$ basis set, Bhatia and Temkin using Feshbach formalism [38] and a 84term Hylleraas for the resonant wave functions, Zhou et al. [26] using hyperspherical close-coupling calculations, and Wintgen and Delande [36] using a complex rotation method. Present results are in agreement with all these theoretical works using highly accurate calculations.

In Table 3, present results on $\mathrm{Li}^{+}$are given along with the theoretical works of Zhou et al. [26], Sanchez and Martin [56], Bhatia and Temkin [38], Diehl et al. [57], and with the experimental works of Carroll and Kennedy [52] and Scully et al. [53]. For index profile, our result is in good agreement with the experimental ones but is greater than the other theoretical works. Discrepancies observed could be explained by the fact that the nonresonant Cross-Section we calculated is greater than those of other theoretical results. The present correlation coefficient is in good agreement with the only available one. Moreover, for better comparison, it would be necessary to get more experimental results.

In Table 4, the comparison of our results on $\mathrm{C}^{4+}$ is made with the only available theoretical work of Zhou et al. [26]. Any experimental work has been reported in the literature. We noticed also the greater values of our profile index and nonresonant Cross-Section. In Table 5, we present our results concerning the $\mathrm{N}^{5+}, \mathrm{O}^{6+}$, and $\mathrm{Ne}^{8+}$ Ions without any comparison because of lack of other experimental and theoretical results.

The analysis of the different parameters show that the nonresonant Cross-Section decreases with increasing $Z$ as found by Zhou et al. [26]. This decreasing is due to the fact that the matrix elements which contain the wave function of the ground state decrease with the electron-electron interaction with increasing $Z$. For all considered ions, the correlation coefficient $\rho^{2}$ is equal or very close to unity, which is expected from (15) when there is one open channel. For profile index, the behaviour is difficult to explain. It increases with $Z$, from -2.86 for $\mathrm{He}$, to -1.5 for $\mathrm{Li}^{+}$, and -1.20 for 
$\mathrm{C}^{4+}$, thereafter it decreases from -1.22 for $\mathrm{N}^{5+}$ to -1.38 for $\mathrm{Ne}^{8+}$. This disagrees with the analysis of Sanchez and Martin [56] who found a limit of -1.7 by increasing $Z$. However, the unique remark is the negative sign of $q$ for all ions which agree with the Fano and Cooper [59] analysis. Thus, as already noticed by Morgan and Ederer [3], the profile index $q$ is the most parameter to measure and to calculate, larger variation in calculations and experiments is observed.

\section{Conclusion}

In this work, nonresonant Cross-Section, correlation coefficient and profile index of the photoionization of helium and heliumlike $\mathrm{Li}^{+}, \mathrm{C}^{4+}, \mathrm{N}^{5+}, \mathrm{O}^{6+}$, and $\mathrm{Ne}^{8+}$ under the $n=2$ threshold have been calculated. For helium, a good agreement has been found between our $q$ values and the experimental and theoretical values. For $\mathrm{Li}^{+}$, our result for $q$ is close to the experimental work, but greater than the other theoretical values as for $\mathrm{C}^{4+}$. The analysis shows that it will be necessary to determine these parameters for all the Rydberg series under the $n=2$ threshold and also for states lying under the upper thresholds when several channels are open for a better understanding of their behaviour. More experimental values are requested for a better understanding of these discrepancies. For the nonresonant Cross-Sections and correlation parameter, quite good agreement was found between present results and those previously obtained with the use of highly accurate calculations.

\section{Acknowledgments}

The authors would like to thank the Third world Academy of Sciences (TWAS) and the Abdus Salam International Centre of Theoretical Physics for their financial support. The authors also express our recognition to the late Professor Denardo.

\section{References}

[1] R. P. Madden and K. Codling, "New autoionizing atomic energy levels in He, Ne, and Ar," Physical Review Letters, vol. 10, no. 12 , pp. 516-518, 1963.

[2] P. R. Woodruff and J. A. R. Samson, "Measurements of partial cross sections and autoionization in the photoionization of helium to $\mathrm{He}^{+}(\mathrm{N}=2)$," Physical Review A, vol. 25, no. 2, pp. 848-856, 1982.

[3] H. D. Morgan and D. L. Ederer, "Photoionization cross section of helium for photon energies 59-67 eV: the $(s p, 2 n+)^{1} P^{o}$ Rydberg series of autoionizing resonances," Physical Review A, vol. 29, no. 4, pp. 1901-1906, 1984.

[4] D. W. Lindle, T. A. Ferrett, P. A. Heimann, and D. A. Shirley, "Complete photoemission study of the $\mathrm{He} 1 \mathrm{~s}^{2} \rightarrow 3 \mathrm{~s} 3 \mathrm{p}$ autoionizing resonance," Physical Review A, vol. 36, no. 5, pp. 2112-2119, 1987.

[5] H. Kossmann, B. Krässig, and V. Schmidt, "New determination of Beutler-Fano parameters for the $3 \mathrm{~s} 3 \mathrm{p}^{1} \mathrm{P}_{1}$ resonance in helium," Journal of Physics B, vol. 21, no. 9, pp. 1489-1497, 1988.

[6] M. Zubek, G. C. King, P. M. Rutter, and F. H. Read, "Photoionisation studies of autoionising states of helium between 69 and 77 eV," Journal of Physics B, vol. 22, no. 21, pp. 3411-3421, 1989.

[7] M. Zubek, A. G Dawber, R. I. Hall, L. Avaldi, K. Ellis, and G. C. King, "Angular distribution of photoelectrons from ionization of helium into the $\mathrm{He}^{+}(\mathrm{N}=2)$ states, between 69 and $77 \mathrm{eV}$," Journal of Physics B, vol. 24, no. 14, p. L337, 1992.

[8] M. Domke, C. Xue, A. Puschmann et al., "Extensive doubleexcitation states in atomic helium," Physical Review Letters, vol. 66, no. 10, pp. 1306-1309, 1991.

[9] M. Domke, G. Remmers, and G. Kaindl, "Observation of the $(s p, n d)^{1} P^{\circ}$ double-excitation Rydberg series of helium," Physical Review Letters, vol. 69, no. 8, pp. 1171-1174, 1992.

[10] M. Domke, K. Schulz, G. Remmers, A. Gutiérrez, G. Kaindl, and D. Wintgen, "Interferences in photoexcited doubleexcitation series of He," Physical Review A, vol. 51, no. 6, pp. R4309-R4312, 1995.

[11] M. Domke, K. Schulz, G. Remmers, G. Kaindl, and D. Wintgen, "High-resolution study of $\mathrm{P}$ double-excitation states in helium," Physical Review A, vol. 53, no. 3, pp. 1424-1438, 1996.

[12] K. Schulz, G. Kaindl, M. Domke et al., "Observation of new rydberg series and resonances in doubly excited helium at ultrahigh resolution," Physical Review Letters, vol. 77, no. 15, pp. 3086-3089, 1996.

[13] R. Moccia and P. Spizzo, "An $\mathrm{L}^{2}$ calculation of the ${ }^{1,3} \mathrm{P}^{0}$ resonances of atomic helium," Journal of Physics B, vol. 20, no. 7, pp. 1423-1431, 1987.

[14] C. F. Fischer and M. Idrees, "Spline methods for resonances in photoionization cross sections," Journal of Physics B, vol. 23, no. 4 , p. 679, 1990.

[15] T. N. Chang, "He photoionization to the doubly excited (2pnd) and $\left(s p, 2 n^{-}\right)^{1} P^{\circ}$ series," Physical Review A, vol. 47, no. 4, pp. 3441-3443, 1993.

[16] J. Macek, "Properties of autoionizing states of He," Journal of Physics B, vol. 1, no. 5, pp. 831-843, 1968.

[17] C. D. Lin, "Correlations of excited electrons. The study of channels in hyperspherical coordinates," Physical Review A, vol. 10, no. 6, pp. 1986-2001, 1974.

[18] C. D. Lin and J. H. Macek, "Radial and angular correlations of two excited electrons. IV. Comparison of configurationinteraction wave functions with the group-theoretical basis functions," Physical Review A, vol. 29, no. 5, pp. 2317-2321, 1984.

[19] C. D. Lin, "Classification of doubly excited states of twoelectron atoms," Physical Review Letters, vol. 51, no. 15, pp. 1348-1351, 1983.

[20] C. D. Lin, "Hyperspherical coordinate approach to atomic and other Coulombic three-body systems," Physics Report, vol. 257, no. 1, pp. 1-83, 1995.

[21] J. Z. Tang and I. Shimamura, "Mechanism of the enhancement of some high-lying resonance series in the photoionization spectra of excited helium," Physical Review A, vol. 50, no. 2, pp. 1321-1327, 1994.

[22] H. R. Sadeghpour, "Nonadiabatic selection rules for the photoabsorption of $\mathrm{H}^{-}$and He," Physical Review A, vol. 43, no. 11, pp. 5821-5831, 1991.

[23] T. Morishita, K. I. Hino, S. Watanabe, and M. Matsuzawa, "Proton-antiproton impact-electron emission spectra of He: comparison of theory with experiment," Physical Review A, vol. 53, no. 4, pp. 2345-2358, 1996.

[24] D. H. Oza, "Phase shifts and resonances for electron scattering by $\mathrm{He}^{+}$below the $\mathrm{N}=2$ threshold," Physical Review A, vol. 33, no. 2, pp. 824-838, 1986. 
[25] S. Salomonson, S. L. Carter, and H. P. Kelly, "Calculation of helium photoionization with excitation including angular distribution and resonance structure," Physical Review A, vol. 39, no. 10, pp. 5111-5126, 1989.

[26] B. Zhou, C. D. Lin, J.-Z. Tang, S. Watanabe, and M. Matsuzawa, "A hyperspherical close-coupling calculation of photoionization from the $\mathrm{He}$ atom, $\mathrm{Li}^{+}$and $\mathrm{C}^{+}$ions. I. Below the $\mathrm{N}=2$ threshold," Journal of Physics B, vol. 26, no. 16, p. 2555, 1993.

[27] K. A. Berrington, P. G. Burke, W. C. Fon, and K. T. Taylor, "Photoionisation of helium," Journal of Physics B, vol. 15, no. 17, pp. L603-L608, 1982.

[28] J. Yan, Y.-Z. Qu, L. Voky, and J.-M. Li, "Polarization effect on He doubly excited states below the $\mathrm{N}=2$ threshold of $\mathrm{He}^{+}$," Physical Review A, vol. 57, no. 2, pp. 997-1005, 1998.

[29] T. W. Gorczyca, J. E. Rubensson, C. Såthe et al., "Radiative and relativistic effects in the decay of highly excited states in helium," Physical Review Letters, vol. 85, no. 6, pp. 1202-1205, 2000.

[30] Y. Jiang, J. Yan, J. Li, J. Sun, and L. Wan, "Photoionization of helium between the $\mathrm{N}=2$ and $\mathrm{N}=5$ thresholds of $\mathrm{He}^{+}$: partial differential cross sections," Physical Review A, vol. 61, no. 3, Article ID 032721, 7 pages, 2000.

[31] H. W. van der Hart and C. H. Greene, "Regularities and irregularities in partial photoionization cross sections of He," Physical Review A, vol. 66, no. 2, Article ID 022710, 8 pages, 2002.

[32] T. Schneider, C.-N. Liu, and J.-M. Rost, "Intermanifold similarities in partial photoionization cross sections of helium," Physical Review A, vol. 65, no. 4, Article ID 042715, 9 pages, 2002.

[33] Y. K. Ho, "Complex-coordinate calculations for doubly excited states of two-electron atoms," Physical Review A, vol. 23, no. 5, pp. 2137-2149, 1981.

[34] Y. K. Ho, "Resonance positions and widths of doubly excited $\mathrm{N}^{5+}$ ions associated with $\mathrm{N}=3,4$, and $5 \mathrm{~N}^{6+}$ thresholds," Physical Review A, vol. 35, no. 5, pp. 2035-2043, 1987.

[35] Y. K. Ho, "High-lying doubly excited ${ }^{1} P^{\circ}$ states of $\mathrm{H}^{-}$," Physical Review A, vol. 45, no. 1, pp. 148-153, 1992.

[36] D. Wintgen and D. Delande, "Double photoexcitation of ${ }^{1} P^{\circ}$ states in helium," Journal of Physics B, vol. 26, no. 14, p. L399, 1993.

[37] A. K. Bhatia and A. Temkin, "Calculation of autoionization of $\mathrm{He}$ and $\mathrm{H}^{-}$using the projection-operator formalism," Physical Review A, vol. 11, no. 6, pp. 2018-2024, 1975.

[38] A. K. Bhatia and A. Temkin, "Line-shape parameters for P1 Feshbach resonances in $\mathrm{He}$ and $\mathrm{Li}^{+}$," Physical Review A, vol. 29, no. 4, pp. 1895-1900, 1984.

[39] H. Bachau, "Position and widths of autoionising states in the helium isoelectronic sequence above the $\mathrm{N}=2$ continuum," Journal of Physics B, vol. 17, no. 9, pp. 1771-1784, 1984.

[40] A. Macías, F. Martín, A. Riera, and M. Yánez, "Simple discretization method for autoionization widths. II. Atoms," Physical Review A, vol. 36, no. 9, pp. 4187-4202, 1987.

[41] V. V. Balashov, S. I. Grishanova, I. M. Kruglova, and V. S. Shenashenko, "Resonance photoionizationof helium and helium-like ions," Optics and Spectroscopy, vol. T28, no. 3, pp. 466-471, 1970, English Translation from Opt. Spektrosk, vol. 28. pp. 859-868, 1970.

[42] A. Wagué, "Application of the diagonalization approximation to the $\mathrm{n}=2$ resonant photoionization of helium-like systems," Zeitschrift für Physik D, vol. 6, no. 4, pp. 337-344, 1987.
[43] N. A. B. Faye and A. Wagué, "Doubly-excited state resonances of the helium-like nitrogen ion $\mathrm{N}^{5+}$ near the $\mathrm{n}=3$ threshold of $\mathrm{N}^{6+}$," Zeitschrift für Physik D, vol. 31, no. 1, pp. 37-40, 1994.

[44] N. A. B. Faye and A. Wague, "Resonant photoionization of $\mathrm{O}^{6+}$ below the $\mathrm{n}=2$ threshold of the reduced ion," Journal of Physics B, vol. 28, no. 9, pp. 1735-1742, 1995.

[45] A. S. Ndao, A. Wagué, N. A.B. Faye, and A. Konté, "High-lying ${ }^{1,3} \mathrm{P}^{0}$ resonance states of $\mathrm{He}(\mathrm{n}=4)$ and $\mathrm{H}^{-}(\mathrm{n}=5)$, , European Physical Journal D, vol. 5, no. 3, pp. 327-334, 1999.

[46] M. Biaye, A. Konté, A. S. Ndao, N. A. B. Faye, and A. Wagué, "The singlet doubly excited $(n l)^{2}$ states of helium-like systems," Physica Scripta, vol. 71, no. 1, pp. 39-42, 2005.

[47] N. A. B. Faye, A. S. Ndao, A. Konté, M. Biaye, and A. Wague, "Energy levels in the resonant photoionization of heliumlike $\mathrm{Ne}^{8+}$," Physical Review A, vol. 75, no. 4, Article ID 042713, 5 pages, 2007.

[48] G. Tanner, K. Richter, and J. M. Rost, "The theory of two-electron atoms: between ground state and complete fragmentation," Reviews of Modern Physics, vol. 72, no. 2, pp. 497-544, 2000.

[49] Z.-S. Yuan, X.-Y. Han, X.-J. Liu et al., "Theoretical investigations on the dynamical correlation in double excitations of helium by the R-matrix method," Physical Review A, vol. 70, no. 6, Article ID 062706, 2004.

[50] X. W. Fan and K. T. Leung, "Momentum-transfer dependence of Fano parameters for the $\left(1 s^{2}\right)^{1} \mathrm{~S} \rightarrow(2 \mathrm{~s} 2 \mathrm{p})^{1} \mathrm{P}$ doubly excited transition in helium," Journal of Physics B, vol. 34, no. 5, pp. 811-818, 2001.

[51] K. C. Prince, R. Richter, M. de Simone, M. Alagia, and M. Coreno, "Photoabsorption cross section and ion-yield spectra of helium double-excitation resonances," Physical Review A, vol. 68, no. 4, Article ID 044701, 4 pages, 2003.

[52] P. K. Carroll and E. T. Kennedy, "Doubly excited autoionization resonances in the absorption spectrum of $\mathrm{Li}^{+}$formed in a laser-produced plasma," Physical Review Letters, vol. 38, no. 19, pp. 1068-1071, 1977.

[53] S. W. J. Scully, I. Álvarez, C. Cisneros et al., "Doubly excited resonances in the photoionization spectrum of $\mathrm{Li}^{+}$," Journal of Physics: Conference Series, vol. 58, no. 1, pp. 387-390, 2007.

[54] P. L. Altick and E. N. Moore, "Configuration interaction in the helium continuum," Physical Review, vol. 147, no. 1, pp. 5965, 1966.

[55] P. G. Burke and D. D. McVicar, "Resonances in e-He scattering and the photoionization of He," Proceedings of the Physical Society, vol. 86, no. 5, pp. 989-1006, 1965.

[56] I. Sanchez and F. Martin, "Photoionization of He-like systems below the $\mathrm{N}=2$ threshold " Journal of Physics B, vol. 23, no. 23, p. 4263, 1990.

[57] S. Diehl, D. Cubaynes, J. M. Bizau et al., "New high-resolution measurements of doubly excited states of $\mathrm{Li}^{+}$," Journal of Physics B, vol. 32, no. 17, pp. 4193-4207, 1999.

[58] U. Fano, "Effects of configuration interaction on intensities and phase shifts," Physical Review, vol. 124, no. 6, pp. 18661878, 1961.

[59] U. Fano and J. W. Cooper, "Line profiles in the far-uv absorption spectra of the rare gases," Physical Review, vol. 137, no. 5, pp. A1364-A1379, 1965. 

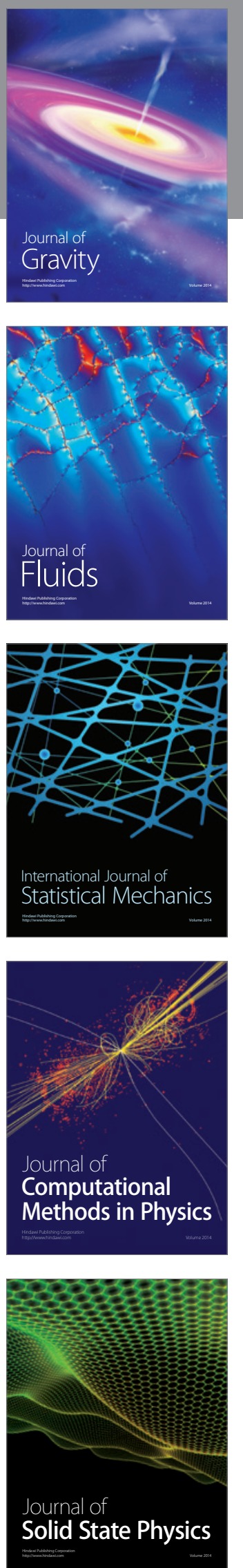

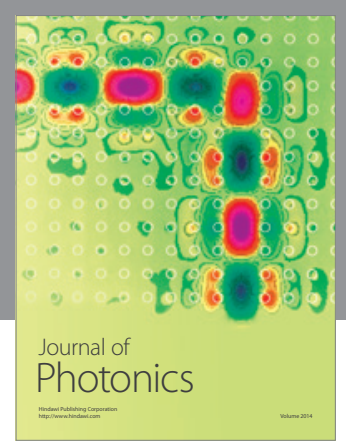

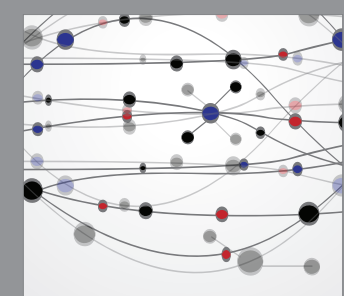

The Scientific World Journal
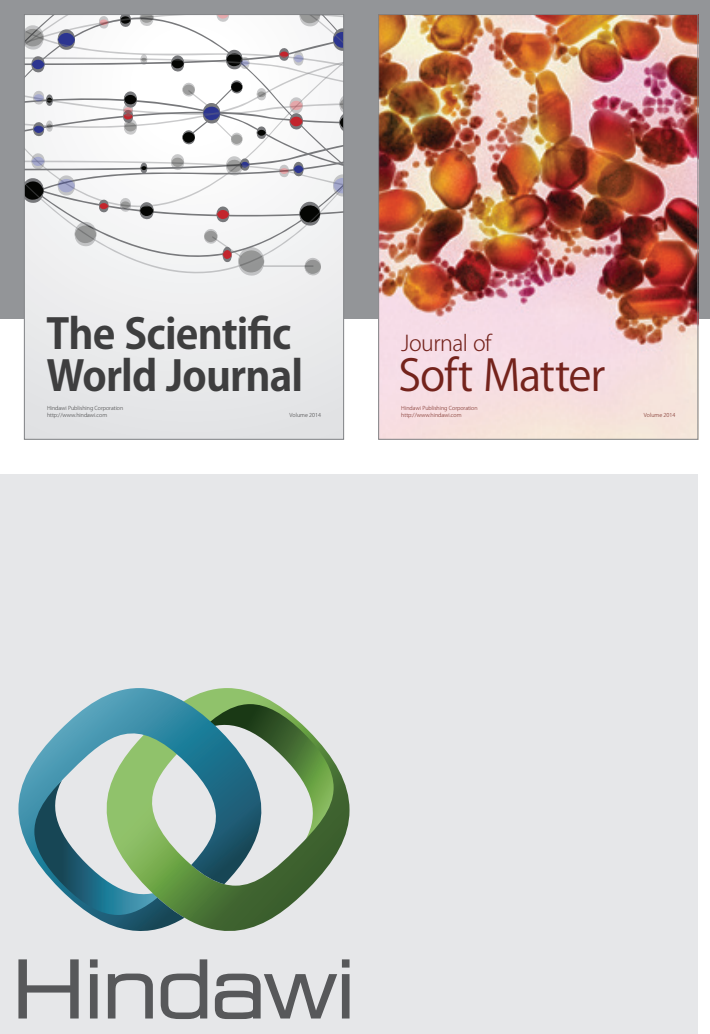

Submit your manuscripts at

http://www.hindawi.com
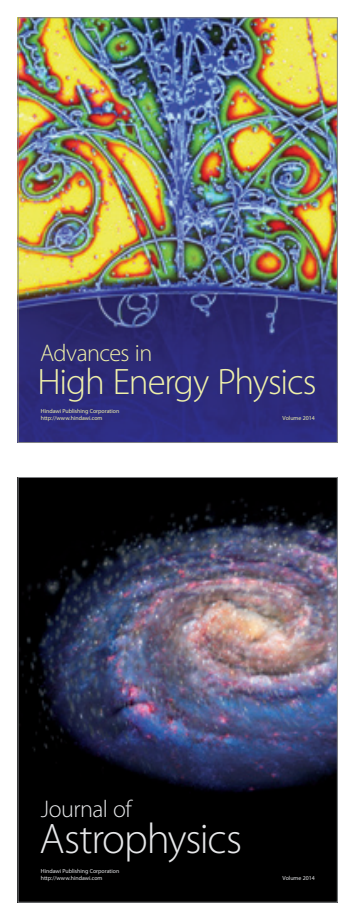
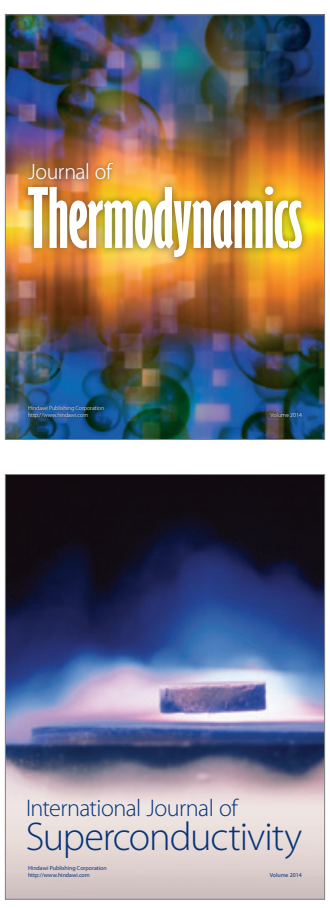
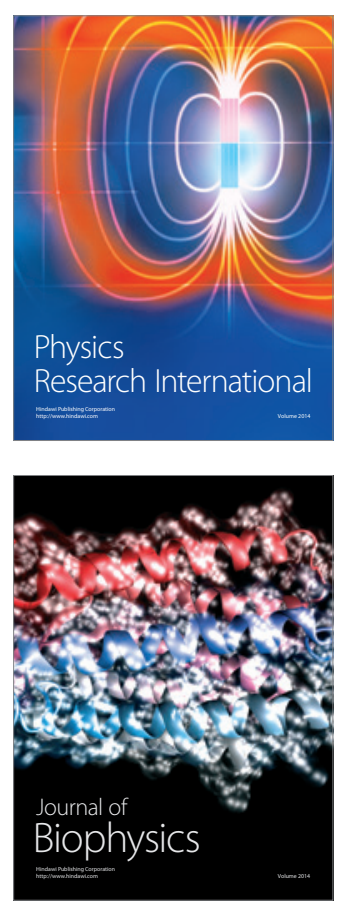
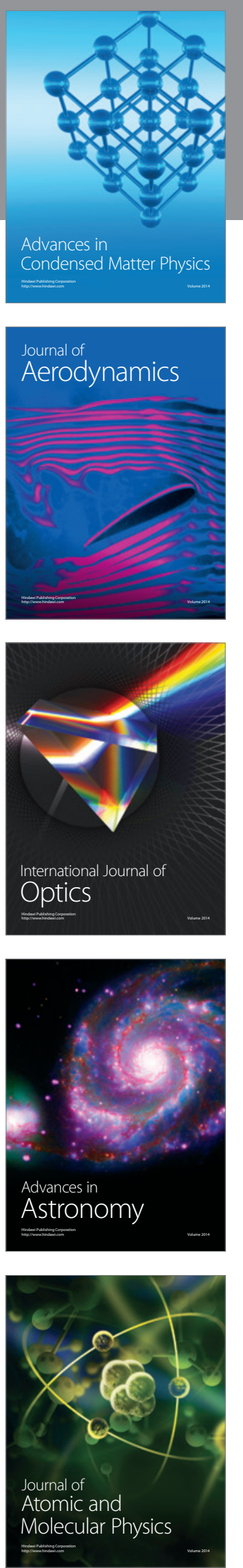